\title{
FLARE-PRODUCED CORONAL WAVES
}

\author{
FRIEDRICH MEYER \\ (Max-Planck-Institut für Physik und Astrophysik, München, Germany)
}

\begin{abstract}
A BSTRA C T
We present a coronal propagation model for fast chromospheric disturbances following flares that were first observed by Moreton and Ramsey, and deduce estimates of average coronal magnetic fields (6 gauss) and disturbance energies $\left(\leqslant 10^{29} \mathrm{erg}\right)$.
\end{abstract}

Flare-produced hydromagnetic waves were first observed by Moreton $(1960,1965)$ and Ramsey as chromospheric disturbances on the wings of the $\mathrm{H} \alpha$ line. They travel across the solar disk with speeds of the order of $1000 \mathrm{~km} / \mathrm{sec}$, can excite filaments to oscillations and trigger other flares.

In the event of September 20, 1963 observed by Moreton and Ramsey (Moreton, 1965 ; cf. also Zirin, 1966, p. 400ff.), such a wave apparently was caused by the sudden brightening of the flare. It propagated away from its origin with a nearly constant speed of $750 \mathrm{~km} / \mathrm{sec}$ into an angular sector of about $60^{\circ}$ covering a distance of several hundred thousand kilometers. The widths of the disturbance was about $100000 \mathrm{~km}$, and the $\mathrm{H} \alpha$ Doppler shift indicated a material motion of about $10 \mathrm{~km} / \mathrm{sec}$ away from the observer followed by a recovery in the opposite direction.

The high constant propagation speed and the low wave amplitude of this event are incompatible with a chromospheric propagation. For a temperature of $1.6 \times 10^{4}{ }^{\circ} \mathrm{K}$ the chromospheric sound velocity is $18 \mathrm{~km} / \mathrm{sec}$ and for a density of $3 \times 10^{10} / \mathrm{cm}^{3}$ and a 5 gauss magnetic field the chromospheric Alfvén velocity is about $55 \mathrm{~km} / \mathrm{sec}$. The $750 \mathrm{~km} / \mathrm{sec}$ wave then would have moved with a Mach number of the order of 10 . A shock wave of such strength would have shown large wave amplitudes (of the order of the propagation speed) and strong dissipation, i.e. slowing down. Both such effects are not observed.

These difficulties disappear if a coronal propagation is considered.* In the corona the sound and Alfvén speeds both are about a factor of 10 higher than in the chromosphere. This results from a coronal temperature roughly 100 times larger and a coronal density 100 times smaller than their corresponding chromospheric values.

In the corona the large ratio of the magnetic to the internal gas-energy density clearly separates the 3 hydromagnetic modes. This ratio is 10 at the bottom of the corona and increases upwards (for a magnetic field $B=5$ gauss, a temperature of

* The coronal propagation of such disturbances was independently suggested by H. U. Schmidt (private communication). 
1.6 million degrees and a particle density of $3 \times 10^{8} / \mathrm{cm}^{3}$ ). The slow mode is reduced to a sound-wave traveling along the guiding magnetic field. The incompressible Alfvén mode moves with Alfvén speed $V_{\mathrm{A}}=B /(4 \pi \rho)^{1 / 2}$ (where $\rho$ is the mass density) but is also confined to propagation along the fieldlines. The fast hydromagnetic mode moves also at Alfvén speed (slightly modified by the sound velocity) but can propagate in all directions with respect to the magnetic field. It is this latter mode that has to be made responsible for the fast disturbances spreading across the solar disk.

The propagation of this mode in the corona is strongly influenced by downward refraction in the coronal density gradient and upward reflection at the chromospheric interface. The refraction is caused by the density-dependent increase of the Alfven speed with height and prevents the energy from escaping upwards. The sudden density increase between corona and chromosphere produces the almost complete reflection. Only a small fraction of the energy penetrates into the chromosphere and causes the observed $\mathrm{H} \alpha$-motions. The propagation is further influenced by dispersion which must finally disintegrate any disturbance into wavetrains of different speed. Waves of long horizontal extent reach higher up where the Alfvén speed is larger. They therefore travel faster than small-scale waves.

For a quantitative comparison we simplify the corona to an isothermal gas of 1.6 million degrees lying above a chromosphere a hundred times cooler and denser. For simplicity we first investigate the case when the magnetic field is vertical and constant (Figure 1). The first approximation consists in neglecting the small chromospheric penetration altogether and assuming a perfectly reflecting interface.

As is suggested by the dispersion we consider the individual harmonic waves which together make up a wave packet.

For each value of the horizontal wave number $k$ there exists a discrete set of heightdependent eigenfunctions with $n=0,1,2, \ldots$ nodes (the vertical set is discrete rather than continuous because of the exponential increase of the signal velocity $V_{\mathrm{A}}$ with

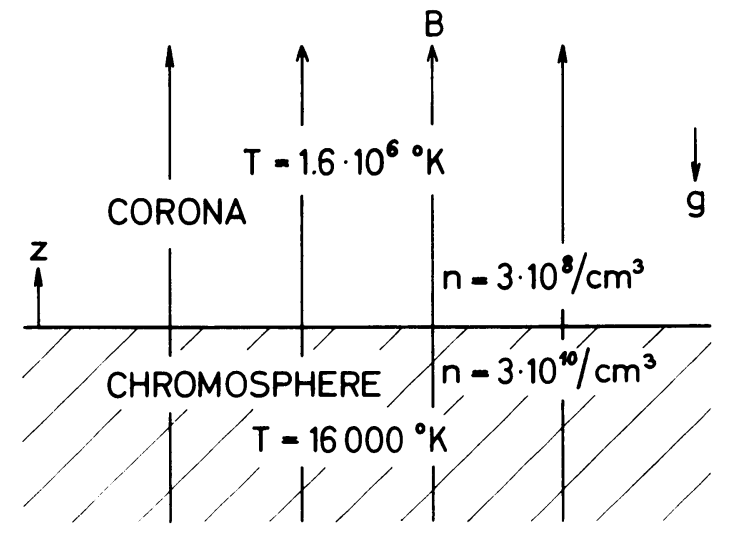

FIG. 1. The corona-chromosphere model. 
height). To each wave $(k, n)$ belongs an eigenfrequency $\omega=\omega_{n}(k)$. From this one determines the phase velocity $V_{\mathrm{ph}}=\omega / k$ and the group velocity $V_{\mathrm{gr}}=\mathrm{d} \omega / \mathrm{d} k$. Figure 2 gives the result of such an evaluation in form of a dispersion diagram. It shows the nine lowest eigenfrequencies as a function of $k$. The six horizontal lines belong to Alfvén modes. Their group velocity is zero since they cannot propagate across the magnetic field. The three sloping lines belong to the fast mode with a remarkably

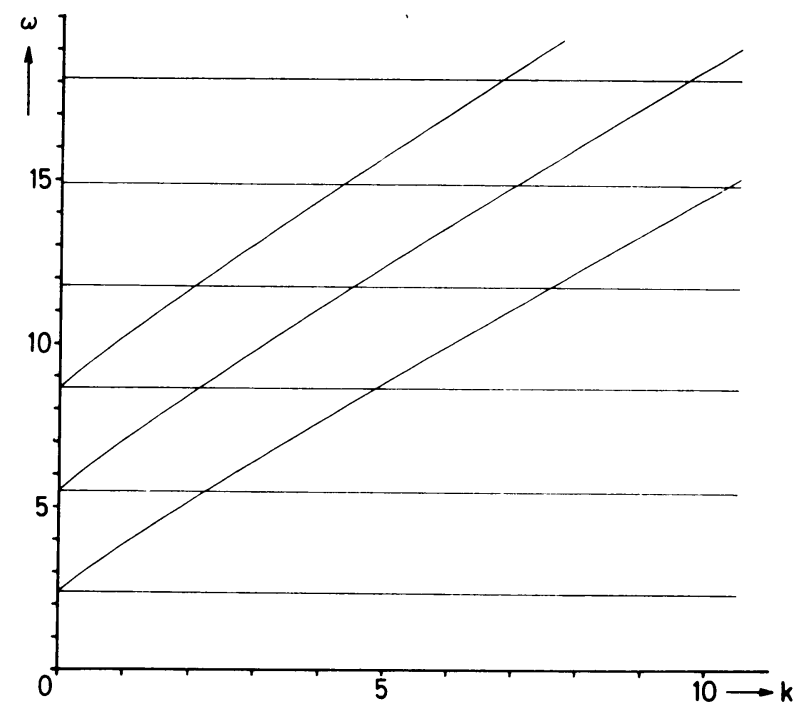

FIG. 2. Angular frequency $\omega$ over horizontal wave number $k$ in the corona. The magnetic field is vertical. Unit of length is twice the coronal density-scale height. Unit of time is the unit of length divided by the Alfvén speed at the bottom of the corona.

constant group velocity. In Figure 3 we plot phase and group velocity as a function of $1 / k$. The small variation of the group velocity in the range of the observed dimensions $(\approx 3 \leqslant k \leqslant 10)$ will account for the long distance across the Sun over which the disturbance on the September 20, 1963 event could be followed.

Equating the observed velocity of this disturbance to the group velocity for the characteristic wave number $(k \approx 7)$ one can determine the physical value of the unit velocity, i.e. the Alfven speed at the bottom of the corona. With a density of $3 \times 10^{8} / \mathrm{cm}^{3}$ this leads to a coronal magnetic field of 6 gauss. It is an average field over the dimension of the wave. The value depends only on the square root of the assumed coronal density. Though earlier determinations of undisturbed photospheric fields suggested lower values of the coronal field, the 6 gauss may be reasonable in view of recent observations. They found strong photospheric flux concentrations that should at least partially spread out into the corona.

The influence of an inclined magnetic field on the propagation of such coronal 


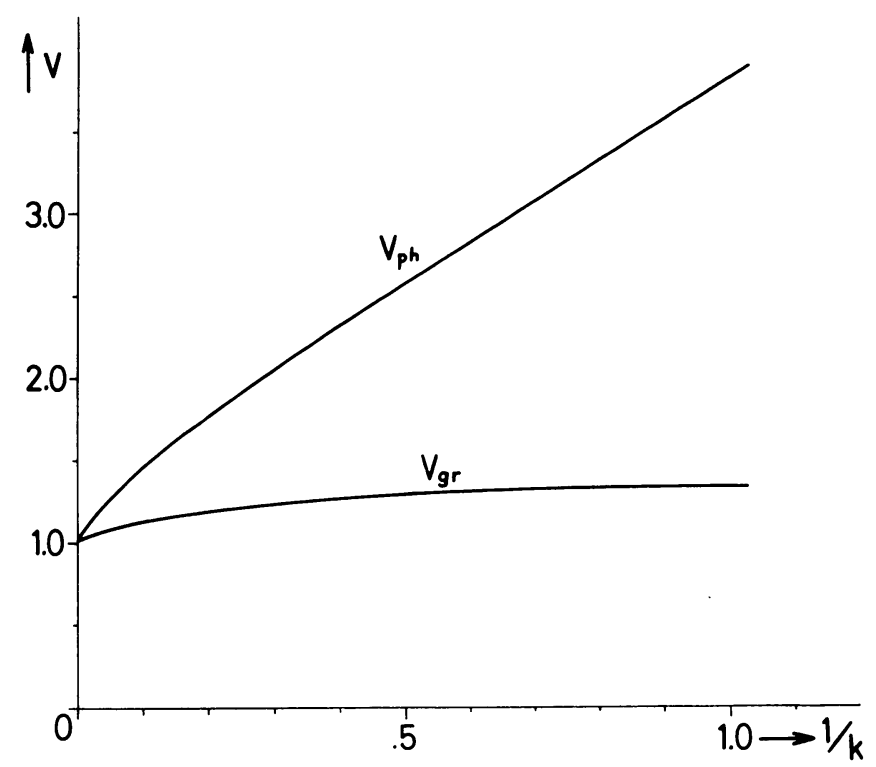

FIG. 3. Phase velocity $V_{p h}$ and group velocity $V_{g r}$ as a function of $1 / k$ for the lowest order fast mode $(n=0)$. Units as in Figure 2.

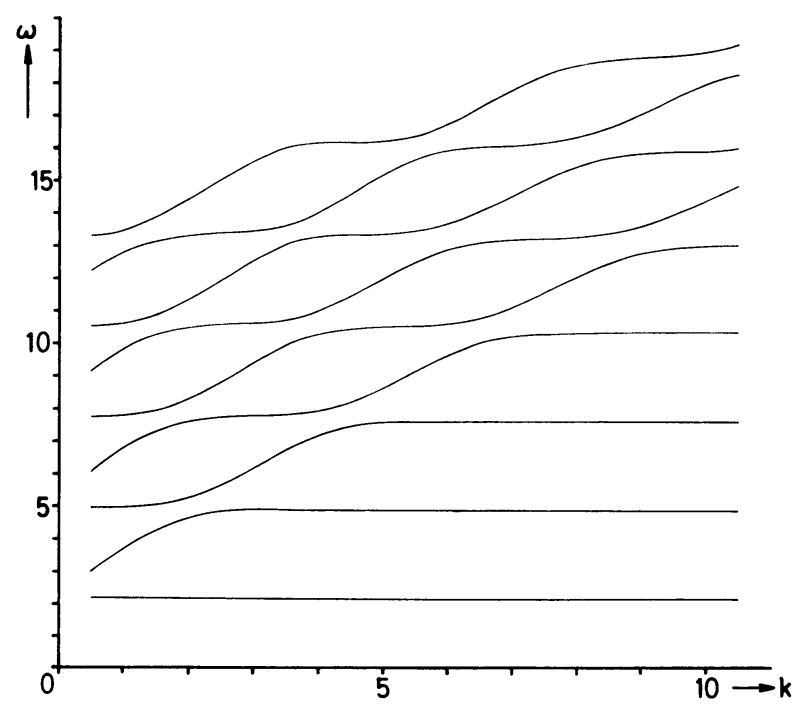

FIG. 4. Dispersion diagram for a magnetic field inclined $30^{\circ}$ to the vertical. Direction of horizontal wave vector at $45^{\circ}$ to the horizontal magnetic-field component. Units as in Figure 2. 
disturbances was investigated together with E. Meyer-Hofmeister on a recent visit to the Sacramento Peak Observatory. We found that fast modes are not changed if they propagate in the direction of the horizontal field component. Alfvén modes for this case have lowered eigenvalues. This is due to an increased 'optical path' along the inclined fieldlines.

For other directions of this horizontal propagation fast and Alfvén modes are superposed to give the eigenfunctions. This is caused by the lower boundary at which fast modes on reflection also excite Alfvén modes, and vice versa. The resulting mixture of fast and Alfvén modes in each eigenfunction changes with the wave number $k$. This leads to the characteristic frequency variation for the eigenmodes in Figure 4. That dispersion diagram is an example for general propagation in an inclined magnetic field. The change of character expresses itself in the group velocity which for a particular eigenvalue varies from near zero values of the Alfvén mode to high values of the fast mode. Thus in inclined fields the dispersion becomes very strong for all directions except those close to the direction of inclination. From this effect we expect an apparent concentration of observed disturbances along that preferred direction. For the September 20, 1963 event such a relation is indicated. A surge preceding the wave phenomenon pointed in projection against the disk in a direction which coincided with that of the later wave propagation. Since the surge had to follow the magnetic field we conclude that it also was tilted in the same direction.

One can determine amplitudes of the coronal waves from the observed chromospheric motions. To do this one has to relax the condition of perfect reflection at the interface and to supplement the coronal pattern by a chromospheric part. This changes the coronal propagation very little. A small imaginary part of the eigenvalues of about $1 \%$ is introduced by the damping which is caused by the energy loss into the chromosphere. The amplitudes of the corona are now connected with those in the chromosphere. For the case of a vertical magnetic field this next order approximation has been performed. For the September 20,1963 event we infer a coronal amplitude maximum of about $200 \mathrm{~km} / \mathrm{sec}$. It is about $\frac{1}{4}$ of the local Alfvén speed. A check on this estimate comes from filament oscillations caused by such coronal waves. The theoretical prediction agrees in the order of magnitude with the observed oscillation amplitudes. The energy contained in the observed disturbance is estimated from this amplitude as $\leqslant 10^{29} \mathrm{erg}$ which is a small fraction of the total flare energy.

\section{References}

Moreton, G.E., (1960) Astronom. J., 65, 494.

Moreton, G.E., (1965) in Stellar and Solar Magnetic Fields. I.A.U. Symp. 22, North-Holland Publishing Co., Amsterdam, p. 371.

Zirin, H. (1966) The Solar Atmosphere, Blaisdell Publishing Co., Waltham, Mass., p. 400ff, Fig.

13.11 and 13.12 . 